\title{
Solutions to Uncertain Volterra Integral Equations by Fitted Reproducing Kernel Hilbert Space Method
}

\author{
Ghaleb Gumah, ${ }^{1}$ Khaled Moaddy, ${ }^{2}$ Mohammed Al-Smadi, ${ }^{3}$ and Ishak Hashim ${ }^{4,5}$ \\ ${ }^{1}$ Faculty of Engineering Technology, Al-Balqa Applied University, Amman 11942, Jordan \\ ${ }^{2}$ Department of Mathematics, Faculty of Science and Arts, Shaqra University, Shaqra 11691, Saudi Arabia \\ ${ }^{3}$ Department of Applied Science, Ajloun College, Al-Balqa Applied University, Ajloun 26816, Jordan \\ ${ }^{4}$ School of Mathematical Sciences, Universiti Kebangsaan Malaysia, 43600 Bangi, Selangor, Malaysia \\ ${ }^{5}$ Research Institute, Center for Modeling \& Computer Simulation (RI/CM \& CS), King Fahd University of Petroleum \& Minerals, \\ Dharan 31261, Saudi Arabia \\ Correspondence should be addressed to Khaled Moaddy; moaddy@yahoo.com
}

Received 11 February 2016; Accepted 8 May 2016

Academic Editor: Leszek Olszowy

Copyright ( 2016 Ghaleb Gumah et al. This is an open access article distributed under the Creative Commons Attribution License, which permits unrestricted use, distribution, and reproduction in any medium, provided the original work is properly cited.

We present an efficient modern strategy for solving some well-known classes of uncertain integral equations arising in engineering and physics fields. The solution methodology is based on generating an orthogonal basis upon the obtained kernel function in the Hilbert space $W_{2}^{1}[a, b]$ in order to formulate the analytical solutions in a rapidly convergent series form in terms of their $\alpha$-cut representation. The approximation solution is expressed by $n$-term summation of reproducing kernel functions and it is convergent to the analytical solution. Our investigations indicate that there is excellent agreement between the numerical results and the RKHS method, which is applied to some computational experiments to demonstrate the validity, performance, and superiority of the method. The present work shows the potential of the RKHS technique in solving such uncertain integral equations.

\section{Introduction}

Building mathematical model of a specific phenomenon under uncertainty is essentially important for a large number of applications in economics, medicine, mathematics, physics, and engineering [1-5]. The concepts of uncertain integral equations have developed in recent years as a new branch of fuzzy mathematics. The uncertain integral equations are powerful tools to introduce uncertain parameters and to deal with their dynamical systems in natural fuzzy environments. They are actually of great importance in the fuzzy analysis theory and its application in fuzzy control models, atmosphere, artificial intelligence, measure theory, quantum optics, and so forth [6-10]. The experts in such areas extensively use these equations to make the uncertain problems, which are usually too complex to be defined in precise terms, more understandable. In many situations, information for real scientific and technological processes is provided under uncertainty, which may arise in the experiment part, data collection, and measurement process as well as when determining the initial values. In classical mathematics, however, crisp equations cannot cope with these situations. Therefore, it is necessary to have some mathematical apparatus to understand this uncertainty. Thus, it is immensely important to develop appropriate and applicable strategy to accomplish the mathematical construction that would appropriately treat uncertain problems and solve them.

During the last decades, many authors have devoted their attention to study solutions to uncertain integral equations using various numerical and analytical methods. Among these attempts are the homotopy analysis method [11], Adomian decomposition method [12], homotopy perturbation method [13], Lagrange interpolation method [14], differential transform method [15], and other methods [16-18]. The purpose of this paper is to extend the application of the reproducing kernel Hilbert space (RKHS) method to provide 
analytic-numeric solutions for a class of uncertain Volterra integral equations of the second kind in the following form:

$$
x(t)=f(t)+\int_{a}^{t} k(t, s) g(x(s)) d s, \quad t \geq a,
$$

where $k(s, t)$ is continuous arbitrary crisp kernel function, $g$ is linear or nonlinear continuous increasing function, $f$ is a continuous fuzzy-valued function, and $x \in W_{2}^{1}[a, b]$ is an unknown function to be determined. If $f(t)$ is a crisp function, then the solution to (1) is crisp. However, if $f(t)$ is a fuzzy function, then this equation may only process fuzzy solutions. Sufficient conditions for the existence of a unique solution to (1) have been given in [18].

Generally, there exists no method that yields an explicit solution for nonlinear fuzzy Volterra integral equation due to the complexities of uncertain parameters involving these equations. Thus, we need an efficient reliable numerical technique for the solutions to such equations. Anyhow, by using the parametric form of fuzzy numbers, we convert the fuzzy Volterra integral equation into a crisp system of integral equations, which are solved numerically using the RKHS approach. The present method has the following characteristics: first, it is of global nature in terms of the solutions obtained as well as its ability to solve other mathematical, physical, and engineering problems; second, it is accurate and the results can be obtained easily; third, in the utilized method, the global approximation and its derivatives can be established on the whole solution domain; fourth, the method does not require discretization of the variables, and it is not affected by the computation round-off errors and one is not faced with necessity of large computer memory and time. For more details and descriptions about the methodology of the RKHS method including its history and theory, its modification, and its characteristics, we refer to [19-26].

This paper is organized as follows. In the next section, we present some necessary definitions and preliminary results from the fuzzy calculus theory including fuzzy Riemann integrability concept. The procedure for solving fuzzy Volterra integral equation (1) is presented in Section 3. In Section 4, reproducing kernel algorithm is built and introduced in the Hilbert space $W_{2}^{1}[a, b]$. The numerical results are reported to demonstrate the superiority and capability of the proposed method by considering some numerical examples in Section 5. The last section is a brief conclusion.

\section{Preliminaries}

The material in this section is basic in certain sense. For the reader's convenience, we present some necessary definitions and notations from fuzzy calculus theory which will be used throughout the paper. The reader is kindly requested to go through [27-30] in order to know more details about fuzzy calculus and fuzzy differential and integral equations.

Let $S$ be a nonempty set, a fuzzy set $u$ in $S$ is characterized by its membership function $u: S \rightarrow[0,1]$, while $u(s)$ is interpreted as the degree of membership of an element $s$ in the fuzzy set $u$ for each $s \in S$. A fuzzy set $u$ on $\mathbb{R}$ is called convex, if, for each $s, t \in \mathbb{R}$ and $\lambda \in[0,1], u(\lambda s+(1-\lambda) t) \geq$ $\max \{u(s), u(t)\}$; is called normal, if there is $s \in \mathbb{R}$ such that $u(s)=1$; and is called upper semicontinuous, if the set $\{s \in \mathbb{R} \mid u(s)>\alpha\}$ is closed for each $\alpha \in[0,1]$. The support of a fuzzy set $u$ is defined as $\{s \in \mathbb{R} \mid u(s)>0\}$.

Definition 1 (see [28]). A fuzzy number $u$ is a fuzzy subset of $\mathbb{R}$ with normal, convex, and upper semicontinuous membership function of bounded support.

For $0<\alpha \leq 1$, put $[u]_{\alpha}=\{s \in \mathbb{R} \mid u(s) \geq \alpha\}$ and $[u]_{0}=\overline{\{s \in \mathbb{R} \mid u(s)>0\}}$, where $\overline{\{\cdot\}}$ is the closure of $\{\cdot\}$. Then, it is easy to establish that $u$ is a fuzzy number if and only if $[u]_{\alpha}$ is a compact convex subset of $\mathbb{R}$ for each $\alpha \in[0,1]$ and $[u]_{1} \neq \phi[27]$. Thus, if $u$ is a fuzzy number, then $[u]_{\alpha}=$ $[\underline{u}(\alpha), \bar{u}(\alpha)]$, where $\underline{u}(\alpha)=\min \left\{s \mid s \in[u]_{\alpha}\right\}$ and $\bar{u}(\alpha)=$ $\max \left\{s \mid s \in[u]_{\alpha}\right\}$ for each $\alpha \in[0,1]$. Hence, the $\alpha$-level set $[u]_{\alpha}$ is a nonempty compact interval for each $\alpha \in[0,1]$ and any $u \in \mathbb{R}_{F}$, where $\mathbb{R}_{F}$ is the set of fuzzy numbers on $\mathbb{R}$. The notation $[u]_{\alpha}$ is called the $\alpha$-cut representation or parametric form of a fuzzy number $u$. The last description leads to the following characterization theorem to define the parametric form of a fuzzy number $u$ in terms of the endpoint functions $\underline{u}(\alpha)$ and $\bar{u}(\alpha)$.

Theorem 2 (see [27]). Suppose that the functions $\underline{u}, \bar{u}$ : $[0,1] \rightarrow \mathbb{R}$ satisfy the following conditions; first, $\underline{u}$ is a bounded increasing function and $\bar{u}$ is a bounded decreasing function with $\underline{u}(\alpha) \leq \bar{u}(\alpha)$; second, for each $r \in(0,1], \underline{u}$ and $\bar{u}$ are left-hand continuous functions at $\alpha=r$; third, $\underline{u}$ and $\bar{u}$ are right-hand continuous functions at $\alpha=0$. Then, $u$ : $\mathbb{R} \rightarrow[0,1]$ defined by $u(r)=\sup \{\alpha: \underline{u}(\alpha) \leq r \leq$ $\bar{u}(\alpha)\}$ is a fuzzy number with parameterization $[\underline{u}(\alpha), \bar{u}(\alpha)]$. Furthermore, if $u: \mathbb{R} \rightarrow[0,1]$ is a fuzzy number with parameterization $[\underline{u}(\alpha), \bar{u}(\alpha)]$, then the functions $\underline{u}$ and $\bar{u}$ satisfy the aforementioned conditions.

In general, we can represent an arbitrary fuzzy number $u$ by an order pair of functions $(\underline{u}, \bar{u})$ based upon the requirements mentioned in Theorem 2. Frequently, we will write simply $\underline{u}_{\alpha}$ and $\bar{u}_{\alpha}$ instead of $\underline{u}(\alpha)$ and $\bar{u}(\alpha)$, respectively, for each $\alpha \in[0,1]$.

The metric structure on $\mathbb{R}_{F}$ is given by the Hausdorff distance mapping $D: \mathbb{R}_{F} \times \mathbb{R}_{F} \rightarrow \mathbb{R}^{+} \cup\{0\}$ such that $D(u, v)=\sup _{\alpha \in[0,1]} \max \left\{\left|\underline{u}_{\alpha}-\underline{v}_{\alpha}\right|,\left|\bar{u}_{\alpha}-\bar{v}_{\alpha}\right|\right\}$ for arbitrary fuzzy numbers $u$ and $v$. In [31], it has been proved that $\left(\mathbb{R}_{F}, D\right)$ is a complete metric space.

Definition 3 (see [32]). Let $u, v \in \mathbb{R}_{F}$, if there exists $w \in \mathbb{R}_{F}$ such that $u=v+w$, then $w$ is called the $H$-difference (Hukuhara difference) of $u$ and $v$, and it is denoted by $u \odot v$.

It is worth mentioning that the sign $\odot$ stands always for the $H$-difference and $u \Theta v \neq u+(-1) v=u-v$. If the $H$ difference $u \odot v$ exists, then $[u \odot v]_{\alpha}=\left[\underline{u}_{\alpha}-\underline{v}_{\alpha}, \bar{u}_{\alpha}-\bar{v}_{\alpha}\right]$.

For arbitrary fuzzy numbers $u=(\underline{u}(\alpha), \bar{u}(\alpha)), v=$ $(\underline{v}(\alpha), \bar{v}(\alpha))$ and $\lambda \in \mathbb{R}$, we define the addition and scalar multiplication by $\lambda$ as $[u+v]_{\alpha}=[u]_{\alpha}+[v]_{\alpha}=\left[\underline{u}_{\alpha}+\underline{v}_{\alpha}, \bar{u}_{\alpha}+\right.$ $\left.\bar{v}_{\alpha}\right]$ and $[\lambda u]_{\alpha}=\lambda[u]_{\alpha}=\left[\min \left\{\lambda \underline{u}_{\alpha}, \lambda \bar{u}_{\alpha}\right\}, \max \left\{\lambda \underline{u}_{\alpha}, \lambda \bar{u}_{\alpha}\right\}\right]$. Moreover, $u=v$ if and only if $[u]_{\alpha}=[v]_{\alpha}$; that is, $\underline{u}_{\alpha}=\underline{v}_{\alpha}$ and $\bar{u}_{\alpha}=\bar{v}_{\alpha}$. 
Definition 4 (see [27]). Let $F$ be a fuzzy-valued function on a compact convex subset $\Omega$ of $\mathbb{R}$. Then, we say that $F$ is continuous at $t_{0} \in \Omega$ if, for every $\epsilon>0$, there exists $\delta=\delta\left(t_{0}, \epsilon\right)>0$ such that $D\left(F(t), F\left(t_{0}\right)\right)<\epsilon$, for all $t \in \Omega$ with $\left\|t-t_{0}\right\|<\delta$.

Theorem 5 (see [27]). Let $F: \Omega \rightarrow \mathbb{R}_{F}$ be a fuzzyvalued function, where $[F(t)]_{\alpha}=\left[F_{\alpha}(t), \bar{F}_{\alpha}(t)\right]$, and then $F$ is continuous on $\Omega$ if and only if both $\underline{F}_{\alpha}(t)$ and $\bar{F}_{\alpha}(t)$ are continuous on $\Omega$.

For the concept of fuzzy integral, we will define the integral of a fuzzy-valued function using the Riemann integral concept $[27,30]$, which has the advantage of dealing properly with fuzzy IEs, as follows.

Definition 6 (see [30]). Let $F: \Omega \rightarrow \mathbb{R}_{F}$ be continuous fuzzyvalued function. For each partition $\mathscr{P}=\left\{t_{0}^{*}, t_{1}^{*}, \ldots, t_{n}^{*}\right\}$ of $\Omega$ and for arbitrary points $\xi_{k} \in\left[t_{k-1}^{*}, t_{k}^{*}\right], 1 \leq k \leq n$, let $\mathscr{R}_{\mathscr{P}}=\sum_{k=1}^{n} F\left(\xi_{k}\right)\left(t_{k}^{*}-t_{k-1}^{*}\right)$ and $\Delta=\max _{1 \leq k \leq n}\left|t_{k}-t_{k-1}\right|$. Then, the definite integral of $F(t)$ over $\Omega$ is defined by $\int_{\Omega} F(t) d t=$ $\lim _{\Delta \rightarrow 0} \mathscr{R}_{\mathscr{P}}$, provided the limit exists in the metric space $\left(\mathbb{R}_{F}, D\right)$.

Definition 7 (see [30]). Let $F: \Omega \rightarrow \mathbb{R}_{F}$ be continuous fuzzyvalued function, where $[F(t)]_{\alpha}=\left[\underline{F}_{\alpha}(t), \bar{F}_{\alpha}(t)\right]$, and then $\int_{\Omega} F(t) d t$ exists, defined by

$$
\begin{aligned}
{\left[\int_{\Omega} F(t) d t\right]_{\alpha} } & =\int_{\Omega}[F(t)]_{\alpha} d t \\
& =\left[\int_{\Omega} \underline{F}_{\alpha}(t) d t, \int_{\Omega} \bar{F}_{\alpha}(t) d t\right],
\end{aligned}
$$

$$
\alpha \in[0,1]
$$

$\int_{\Omega} F(t) d t \in \mathbb{R}_{F}$, whereas $\underline{F}_{\alpha}(t)$ and $\bar{F}_{\alpha}(t)$ are integrable functions over $\Omega$.

It should be noted that the fuzzy integral can be also defined using the Lebesgue-type approach [28] or the Henstock-type approach [31]. However, if $F$ is continuous function, then all approaches yield the same value and results. Moreover, the representation of the fuzzy integral using Definition 6 is more convenient for numerical calculations and computational mathematics.

\section{Formulation of Fuzzy Volterra Integral Equation}

Formulation of the fuzzy IEs is normally the most important part of the process. It consists of the determination of $\alpha$-cut representation form of nonlinear term $g$, the selection of the integrability type, and the separation of the kernel function $k$. In this section, we study the fuzzy IEs using the concept of Riemann integrability in which the FIEs are converted into equivalent system of crisp integral equations (CIEs). These can be done if the solution is fuzzy function, and consequently the integral must be considered as fuzzy integral.

In order to design RKHS algorithm for solving (1), we set $U(t, s, x(s))=k(t, s) g(x(s))$ and we write the fuzzy function $x(t)$ in terms of its $\alpha$-cut representation form to get that $[x(t)]_{\alpha}=\left[\underline{x}_{\alpha}(t), \bar{x}_{\alpha}(t)\right]$. By considering the parametric form for both sides of the FIE (1), one can get

$$
[x(t)]_{\alpha}=[f(t)]_{\alpha}+\int_{a}^{t}[U(t, s, x(s))]_{\alpha} d s, \quad t \geq a,
$$

where $[f(t)]_{\alpha}=\left[\underline{f}_{\alpha}(t), \bar{f}_{\alpha}(t)\right]$ and $[U]_{\alpha}=\left[\underline{U}_{\alpha}, \bar{U}_{\alpha}\right]$ in which

$$
\begin{aligned}
& \underline{U}_{\alpha}(t, s, x(s))= \begin{cases}k(t, s) g\left(\underline{x}_{\alpha}(s)\right), & k(t, s) \geq 0, \\
k(t, s) g\left(\bar{x}_{\alpha}(s)\right), & k(t, s)<0,\end{cases} \\
& \bar{U}_{\alpha}(t, s, x(s))= \begin{cases}k(t, s) g\left(\bar{x}_{\alpha}(s)\right), & k(t, s) \geq 0, \\
k(t, s) g\left(\underline{x}_{\alpha}(s)\right), & k(t, s)<0 .\end{cases}
\end{aligned}
$$

Therefore, according to the previous results, the FIE (1) can be translated into the following equivalent form:

$$
\begin{aligned}
& \underline{x}_{\alpha}(t)=\underline{f}_{\alpha}(t)+\int_{a}^{t} \underline{U}_{\alpha}(t, s, x(s)) d s, \\
& \bar{x}_{\alpha}(t)=\bar{f}_{\alpha}(t)+\int_{a}^{t} \bar{U}_{\alpha}(t, s, x(s)) d s .
\end{aligned}
$$

Let $x: \Omega \rightarrow \mathbb{R}_{F}$ be continuous fuzzy-valued function. If $x$ satisfies (1), then we say that $x$ is a fuzzy solution to FIE (1). On the other aspect as well, the formulation of (1) together with the characterization Theorem 2 shows us how to deal with numerical solutions to FIEs. We can translate the original fuzzy IE equivalently into system of crisp IEs. In conclusion, one does not need to rewrite the numerical methods for crisp IEs in fuzzy setting, but, instead, we can use the numerical methods directly on the obtained crisp integral system.

\section{Construction of the RKHS Method}

In this section, the formulation of exact and approximate solutions to (1) and the implementation method are given. Initially, we utilize the reproducing kernel concept to construct the Hilbert space $W_{2}^{1}[a, b]$. After that, we construct an orthogonal function system of the space using Gram-Schmidt orthogonalization process. Here, $L^{2}[a, b]=\left\{x: \int_{a}^{b} x^{2}(\eta) d \eta<\right.$ $\infty\}$.

Definition 8 (see [33]). Let $E$ be a nonempty abstract set. A function $K: E \times E \rightarrow \mathbb{R}$ is a reproducing kernel of the Hilbert space $H$ if the following conditions are satisfied: firstly, $K(\cdot, x) \in H$ for each $x \in E$. Secondly, $\langle\phi, K(\cdot, x)\rangle=$ $\phi(x)$ for each $x \in E$ and $\phi \in H$.

The last condition is called "the reproducing property" which means that the value of the function $\phi$ at the point $x$ is reproducing by the inner product of $\phi$ with $K(\cdot, x)$. Indeed, a Hilbert space $H$ of functions on a set $E$ is called a reproducing kernel Hilbert space (RKHS) if there exists a reproducing kernel $K$ of $H$. In functional analysis, RKHS is a Hilbert space of functions in which pointwise evaluation is a continuous linear functional. Equivalently, they are spaces that can be defined by reproducing kernels. 
Definition 9 (see [34]). The inner product space $W_{2}^{1}[a, b]$ defined as $W_{2}^{1}[a, b]=\{x(t) \mid x(t)$ is absolutely continuous real-valued function, $\left.x^{\prime}(t) \in L^{2}[a, b]\right\}$. Meanwhile, the inner product and norm in $W_{2}^{1}[a, b]$ are defined, respectively, by

$$
\langle x(t), y(t)\rangle_{W_{2}^{1}}=x(a) y(a)+\int_{a}^{b} x^{\prime}(\xi) y^{\prime}(\xi) d \xi
$$

and $\|x(t)\|_{W_{2}^{1}}=\sqrt{\langle x(t), x(t)\rangle_{W_{2}^{1}}}$, where $x(t), y(t) \in W_{2}^{1}[a, b]$.

Definition 10 (see [34]). The Hilbert space $W_{2}^{1}[a, b]$ is called a reproducing kernel if, for each fixed $t \in[a, b]$, there exists $R(t, s) \in W_{2}^{1}[a, b]$ such that $\langle x(s), R(t, s)\rangle_{W_{2}^{1}}=x(t)$ for any $x(s) \in W_{2}^{1}[a, b]$ and $s \in[a, b]$.

It is important to mention here that the reproducing kernel function $R$ of a Hilbert space $H$ is unique and the existence of $R$ is due to the Riesz representation theorem, where $R$ completely determines the space $H$.

Theorem 11. The Hilbert space $W_{2}^{1}[a, b]$ is a complete reproducing kernel space and its reproducing kernel function $R_{t}(s)$ is given by

$$
R_{t}(s)= \begin{cases}z_{1}(t)+z_{2}(t) s, & s \leq t \\ z_{3}(t)+z_{4}(t) s, & s>t\end{cases}
$$

Proof. Consider

$$
\left\langle x(t), R_{t}(s)\right\rangle_{W_{2}^{1}}=x(a) R_{t}(a)+\int_{a}^{b} x^{\prime}(\xi) \partial_{\xi} R_{t}(\xi) d \xi,
$$

where $x(t), R_{t}(s) \in W_{2}^{1}[a, b]$.

Through iterative integrations by parts for (8), we obtain that

$$
\begin{aligned}
\int_{a}^{b} x^{\prime}(\xi) \partial_{\xi} R_{t}(\xi) d \xi= & \left.x(t) \partial_{s} R_{t}(s)\right|_{s=a} ^{s=b} \\
& -\int_{a}^{b} x(\xi) \partial_{\xi}^{2} R_{t}(\xi) d \xi
\end{aligned}
$$

Thus, it becomes

$$
\begin{aligned}
\left\langle x(t), R_{t}(s)\right\rangle_{W_{2}^{1}}= & x(a)\left(R_{t}(a)-\partial_{s} R_{t}(a)\right) \\
& +x(b) \partial_{s} R_{t}(b) \\
& -\int_{a}^{b} x(\xi) \partial_{\xi}^{2} R_{t}(\xi) d \xi .
\end{aligned}
$$

If $R_{t}(a)-\partial_{s} R_{t}(a)=0$ and $\partial_{s} R_{t}(b)=0$, then $\left\langle x(t), R_{t}(s)\right\rangle_{W_{2}^{1}}=$ $-\int_{a}^{b} x(\xi) \partial_{\xi}^{2} R_{t}(\xi) d \xi$. For each $t \in[a, b]$, if $R_{t}(s)$ also satisfies

$$
\partial_{s}^{2} R_{t}(s)=\delta(s-t)
$$

then $\left\langle x(t), R_{t}(s)\right\rangle_{W_{2}^{1}}=u(s)$.

For $t \neq s$, the characteristic equation of (11) is given by $\lambda^{2}=0$, and then the characteristic values are $\lambda=0$ with multiplicity 2. So, let the expression of the reproducing kernel function $R_{t}(s)$ be as defined in (7). But, on the other aspect as well, if $R_{t}(s)$ satisfies $R_{t}(t+0)=R_{t}(t-0)$, then, by integrating (11) from $t-\varepsilon$ to $t+\varepsilon$ with respect to $s$ and letting $\varepsilon \rightarrow 0$, we have the jump degree of $\partial_{s} R_{t}(s)$ at $s=t$ such that $\partial_{s} R_{t}(t+$ $0)-\partial_{s} R_{t}(t-0)=-1$. Hence, through the last descriptions, the unknown coefficients of (7) can be obtained.

The unique representation of the reproducing kernel function $R_{t}(s)$ in the space $W_{2}^{1}[0,1]$ is provided in [26] and is given by

$$
R_{t}(s)= \begin{cases}1+s, & s \leq t \\ 1+t, & s>t\end{cases}
$$

The space $W_{2}^{1}[a, b]$ is complete Hilbert space with some special properties. So, all the properties of the Hilbert space will be held. Further, this space possesses some special and better properties which could make some problems solved easier. However, these properties require no more integral computation for some functions, instead of computing some values of a function at some nodes. In fact, this simplification of integral computation not only improves the computational speed but also improves the computational accuracy.

In order to illustrate the analytical solution to the model problem, we consider that $L: W_{2}^{1}[a, b] \rightarrow W_{2}^{1}[a, b]$ is an invertible bounded linear operator such that $L x(t)=x(t)$. Thus, system (5) can be converted into the equivalent form as follows:

$$
\begin{aligned}
& L \underline{x}(t)=F(t, \underline{f}(t), T \underline{x}(t), T \bar{x}(t)), \\
& L \bar{x}(t)=G(t, \bar{f}(t), T \underline{x}(t), T \bar{x}(t)),
\end{aligned}
$$

where $T \underline{x}(t)=\int_{0}^{t} k(t, s) g(\underline{x}(s)) d s, T \bar{x}(t)=\int_{0}^{t} k(t, s) g(\bar{x}(s)) d s$ and $\underline{x}(t), \bar{x}(t), f(t)$, and $\bar{f}(t) \in W_{2}^{1}[a, b]$.

Let $\psi_{i}(t)=L^{*} \Phi_{i}(t)$, where $\Phi_{i}(t)=\left.R_{t}(s)\right|_{s=t_{i}}$, for each $i=1,2, \ldots, L^{*}$, is the conjugate operator of $L$ and $\left\{t_{i}\right\}_{i=1}^{\infty}$ is a countable dense subset on $[a, b]$. Next, the subscript $s$ of $L$ means that the operator $L$ acts on the function of $s$ in which $\psi_{i} \in W_{2}^{1}[a, b]$.

Theorem 12. If $\left\{t_{i}\right\}_{i=1}^{\infty}$ is dense on $[a, b]$, then the orthogonal functions system $\left\{\psi_{i}(t)\right\}_{i=1}^{\infty}$ is a complete system of $W_{2}^{1}[a, b]$ and $\psi_{i}(t)=L_{s} R_{t}(s)_{\left.\right|_{s=t_{i}}}$.

Proof. Clearly, $\psi_{i}(t)=L^{*} \Phi_{i}(t)=\left\langle L^{*} \Phi_{i}(s), R_{t}(s)\right\rangle_{W_{2}^{1}}=\left\langle\Phi_{i}(s)\right.$, $\left.L R_{t}(s)\right\rangle_{W_{2}^{1}}=L_{s} R_{t}(s)_{\left.\right|_{s=t_{i}}} \in W_{2}^{1}[a, b]$. Now, for each fixed $x(t) \in W_{2}^{1}[a, b]$, let $\left\langle x(t), \psi_{i}(t)\right\rangle_{W_{2}^{1}}=0, i=1,2, \ldots$, which means that $\left\langle x(t), \psi_{i}(t)\right\rangle_{W_{2}^{1}}=\left\langle x(t), L^{*} \Phi_{i}(t)\right\rangle_{W_{2}^{1}}=\langle L x(t)$, $\left.\Phi_{i}(t)\right\rangle_{W_{2}^{1}}=L x\left(t_{i}\right)=0$. But since $\left\{t_{i}\right\}_{i=1}^{\infty}$ is dense on $[a, b]$, we must have $L x(t)=0$. It follows that $x(t)=0$ from the existence of $L^{-1}$ and the continuity of $x(t)$. So, the proof of the theorem is complete.

In order to utilize the representation form of analytic and approximate solutions to (1), we will use the Gram-Schmidt 
process that produces an orthonormal sequence $\left\{\bar{\psi}_{i}(t)\right\}_{i=1}^{\infty}$ of the space $W_{2}^{1}[a, b]$ from $\left\{\psi_{i}(t)\right\}_{i=1}^{\infty}$ such that

$$
\bar{\psi}_{i}(t)=\sum_{k=1}^{i} \beta_{i k} \psi_{k}(t), \quad i=1,2, \ldots
$$

where $\beta_{i k}$ are orthogonalization coefficients given as

$$
\begin{aligned}
& \beta_{11}=\frac{1}{\left\|\psi_{1}\right\|}, \\
& \beta_{i j}=\frac{-\sum_{k=j}^{i-1} c_{i k} \beta_{k j}}{\sqrt{\left\|\psi_{i}\right\|^{2}-\sum_{k=1}^{i-1}\left(c_{i k}\right)^{2}}} \quad(j<i), \\
& \beta_{i i}=\frac{1}{\sqrt{\left\|\psi_{i}\right\|^{2}-\sum_{k=1}^{i-1}\left(c_{i k}\right)^{2}}} \quad(i>1)
\end{aligned}
$$

in which $c_{i k}=\left\langle\psi_{i}, \bar{\psi}_{k}\right\rangle_{W_{2}^{1}[a, b]}$.

Theorem 13. If $\left\{t_{i}\right\}_{i=1}^{\infty}$ is dense on $[a, b]$ and $\underline{x}(t), \bar{x}(t) \in$ $W_{2}^{1}[a, b]$ are the exact solutions to system (13), and then $\underline{x}(t)$ and $\bar{x}(t)$ satisfy the following form, respectively:

$$
\underline{x}(t)=\sum_{i=1}^{\infty} \sum_{k=1}^{i} \beta_{i k} F\left(t_{k}, \underline{f}\left(t_{k}\right), T \underline{x}\left(t_{k}\right), T \bar{x}\left(t_{k}\right)\right) \bar{\psi}_{i}(t),
$$

$\bar{x}(t)$

$$
=\sum_{i=1}^{\infty} \sum_{k=1}^{i} \beta_{i k} G\left(t_{k}, \bar{f}\left(t_{k}\right), T \underline{x}\left(t_{k}\right), T \bar{x}\left(t_{k}\right)\right) \bar{\psi}_{i}(t),
$$

and the approximate solutions can be obtained by

$$
\begin{aligned}
& \underline{x}_{n}(t) \\
& =\sum_{i=1}^{n} \sum_{k=1}^{i} \beta_{i k} F\left(t_{k}, \underline{f}\left(t_{k}\right), T \underline{x}_{k-1}\left(t_{k}\right), T \bar{x}_{k-1}\left(t_{k}\right)\right) \\
& \quad \cdot \bar{\psi}_{i}(t), \\
& \bar{x}_{n}(t) \\
& =\sum_{i=1}^{n} \sum_{k=1}^{i} \beta_{i k} G\left(t_{k}, \bar{f}\left(t_{k}\right), T \underline{x}_{k-1}\left(t_{k}\right), T \bar{x}_{k-1}\left(t_{k}\right)\right) \\
& \cdot \bar{\psi}_{i}(t),
\end{aligned}
$$

where $\underline{x}_{0}(t)$ and $\bar{x}_{0}(t)($ fixed $) \in W_{2}^{1}[a, b]$.

Proof. From Theorem 12 and the process of (14), it is obvious that the sequence $\left\{\bar{\psi}_{1}(t), \bar{\psi}_{2}(t), \bar{\psi}_{3}(t), \ldots\right\}$ forms an orthonormal system in $W_{2}^{1}[a, b]$. Since $\underline{x}(t)$ and $\bar{x}(t) \in W_{2}^{1}[a, b]$ are the exact solutions to system (13), then $\underline{x}(t)$ can be expanded to Fourier series in terms of normal orthogonal basis $\left\{\bar{\psi}_{i}(t)\right\}_{i=1}^{\infty}$ in $W_{2}^{1}[a, b]$ as follows:

$$
\underline{x}(t)=\sum_{i=1}^{\infty}\left\langle\underline{x}(t), \bar{\psi}_{i}(t)\right\rangle_{W_{2}^{1}} \bar{\psi}_{i}(t),
$$

and since the space $W_{2}^{1}[a, b]$ is the Hilbert space, then the series $\underline{x}(t)=\sum_{i=1}^{\infty}\left\langle\underline{x}(t), \bar{\psi}_{i}(t)\right\rangle \bar{\psi}_{i}(t)$ is convergent in the norm $\|\cdot\|_{W_{2}^{1}}$. Thus, $\underline{x}(t)$ can be written as

$$
\begin{aligned}
\underline{x}(t) & =\sum_{i=1}^{\infty}\left\langle\underline{x}(t), \bar{\psi}_{i}(t)\right\rangle_{W_{2}^{1}} \bar{\psi}_{i}(t) \\
& =\sum_{i=1}^{\infty}\left\langle\underline{x}(t), \sum_{k=1}^{i} \beta_{i k} \psi_{k}(t)\right\rangle_{W_{2}^{1}} \bar{\psi}_{i}(t) \\
& =\sum_{i=1}^{\infty} \sum_{k=1}^{i} \beta_{i k}\left\langle\underline{x}(t), \psi_{k}(t)\right\rangle_{W_{2}^{1}} \bar{\psi}_{i}(t) \\
& =\sum_{i=1}^{\infty} \sum_{k=1}^{i} \beta_{i k}\left\langle\underline{x}(t), L^{*} \Phi_{k}(t)\right\rangle_{W_{2}^{1}} \bar{\psi}_{i}(t) \\
& =\sum_{i=1}^{\infty} \sum_{k=1}^{i} \beta_{i k}\left\langle L \underline{x}(t), \Phi_{k}(t)\right\rangle_{W_{2}^{1}} \bar{\psi}_{i}(t) \\
& =\sum_{i=1}^{\infty} \sum_{k=1}^{i} \beta_{i k} L \underline{x}\left(t_{k}\right) \bar{\psi}_{i}(t) \\
& =\sum_{i=1}^{\infty} \sum_{k=1}^{i} \beta_{i k} F\left(t_{k}, \underline{f}\left(t_{k}\right), T \underline{x}\left(t_{k}\right), T \bar{x}\left(t_{k}\right)\right) \bar{\psi}_{i}(t) .
\end{aligned}
$$

Similarly, one can get $\bar{x}(t)=\sum_{i=1}^{\infty} \sum_{k=1}^{i} \beta_{i k} G\left(t_{k}, \bar{f}\left(t_{k}\right), T \underline{x}\left(t_{k}\right)\right.$, $\left.T \bar{x}\left(t_{k}\right)\right) \bar{\psi}_{i}(t)$. The approximate solutions can be obtained directly by taking finitely many terms in the series representation for the exact solutions $\underline{x}(t)$ and $\bar{x}(t)$ to (16) and (17). So, the proof of the theorem is complete.

Lemma 14. If $x(t) \in W_{2}^{1}[a, b]$, then there exists $M>0$, such that $\|x\|_{C[a, b]} \leq\|x\|_{W_{2}^{1}[a, b]}$, where $\|x\|_{C[a, b]}=\max _{t \in[a, b]}|x(t)|$.

Theorem 15. Suppose that $\left\|\underline{x}_{n}\right\|_{W_{2}^{1}}$ and $\left\|\bar{x}_{n}\right\|_{W_{2}^{1}}$ are bounded in (18). If $\left\{t_{i}\right\}_{i=1}^{\infty}$ is dense on $[a, b]$ and $F(t, f(t), T \underline{x}(t), T \bar{x}(t))$ and $G(t, \bar{f}(t), T \underline{x}(t), T \bar{x}(t)) \in W_{2}^{1}[a, b] \overline{f o r}$ any $f(t), \bar{f}(t)$, $T \underline{x}(t), T \bar{x}(t) \in W_{2}^{1}[a, b]$, then the approximate solutions $\underline{x}_{n}(t)$ and $\bar{x}_{n}(t)$ in iterative formulas (18) are convergent to the analytic solutions $\underline{x}(t)$ and $\bar{x}(t)$ to system (13) in $W_{2}^{1}[a, b]$ and the exact solutions are expressed as $\underline{x}(t)=\sum_{i=1}^{\infty} A_{i} \bar{\psi}_{i}(t)$, $\bar{x}(t)=\sum_{i=1}^{\infty} B_{i} \bar{\psi}_{i}(t)$, where

$$
\begin{aligned}
& A_{i}=\sum_{k=1}^{i} \beta_{i k} F\left(t_{k}, \underline{f}\left(t_{k}\right), T \underline{x}_{k-1}\left(t_{k}\right), T \bar{x}_{k-1}\left(t_{k}\right)\right), \\
& B_{i}=\sum_{k=1}^{i} \beta_{i k} G\left(t_{k}, \bar{f}\left(t_{k}\right), T \underline{x}_{k-1}\left(t_{k}\right), T \bar{x}_{k-1}\left(t_{k}\right)\right) .
\end{aligned}
$$

Proof. The proof can be divided into two steps. Firstly, we will prove the convergence of $\underline{x}_{n}(t)$ and $\bar{x}_{n}(t)$. From (18), 
we infer that $\underline{x}_{n+1}(t)=\underline{x}_{n}(t)+A_{n+1} \bar{\psi}_{n+1}(t)$ and $\bar{x}_{n+1}(t)=$ $\bar{x}_{n}(t)+B_{n+1} \bar{\psi}_{n+1}(t)$. The orthogonality of $\left\{\bar{\psi}_{i}(t)\right\}_{i=1}^{\infty}$ yields that

$$
\begin{aligned}
\left\|\underline{x}_{n+1}(t)\right\|_{W_{2}^{1}}^{2} & =\left\|\underline{x}_{n}(t)\right\|_{W_{2}^{1}}^{2}+\left(A_{n+1}\right)^{2} \\
& =\left\|\underline{x}_{n-1}(t)\right\|_{W_{2}^{1}}^{2}+\left(A_{n}\right)^{2}+\left(A_{n+1}\right)^{2}=\cdots \\
& =\left\|\underline{x}_{0}(t)\right\|_{W_{2}^{1}}^{2}+\sum_{i=1}^{n+1}\left(A_{i}\right)^{2}, \\
\left\|\bar{x}_{n+1}(t)\right\|_{W_{2}^{1}}^{2} & =\left\|\bar{x}_{n}(t)\right\|_{W_{2}^{1}}^{2}+\left(B_{n+1}\right)^{2} \\
& =\left\|\bar{x}_{n-1}(t)\right\|_{W_{2}^{1}}^{2}+\left(B_{n}\right)^{2}+\left(B_{n+1}\right)^{2}=\cdots \\
& =\left\|\bar{x}_{0}(t)\right\|_{W_{2}^{1}}^{2}+\sum_{i=1}^{n+1}\left(B_{i}\right)^{2} .
\end{aligned}
$$

In another formulation, it holds that $\left\|\underline{x}_{n+1}(t)\right\|_{W_{2}^{1}} \geq\left\|\underline{x}_{n}(t)\right\|_{W_{2}^{1}}$ and $\left\|\bar{x}_{n+1}(t)\right\|_{W_{2}^{1}} \geq\left\|\bar{x}_{n}(t)\right\|_{W_{2}^{1}}$. Due to the condition that $\left\|\underline{x}_{n}(t)\right\|_{W_{2}^{1}}$ and $\left\|\bar{x}_{n}(t)\right\|_{W_{2}^{1}}$ are bounded, then $\left\|\underline{x}_{n}(t)\right\|_{W_{2}^{1}}$ and $\left\|\bar{x}_{n}(t)\right\|_{W_{2}^{1}}$ are convergent as $n \rightarrow \infty$. It implies that $\sum_{i=1}^{\infty}\left(A_{i}\right)^{2}<\infty, \sum_{i=1}^{\infty}\left(B_{i}\right)^{2}<\infty$. This means that $\left\{A_{i}\right\}_{i=1}^{\infty}$ and $\left\{B_{i}\right\}_{i=1}^{\infty} \in l^{2}(i=1,2, \ldots)$. On the one hand, since $\left(\underline{x}_{m}(t)-\right.$ $\left.\underline{x}_{m-1}(t)\right) \perp\left(\underline{x}_{m-1}(t)-\underline{x}_{m-2}(t)\right) \perp \cdots \perp\left(\underline{x}_{n+1}(t)-\underline{x}_{n}(t)\right)$, $\left(\bar{x}_{m}(t)-\bar{x}_{m-1}(t)\right) \perp\left(\bar{x}_{m-1}(t)-\bar{x}_{m-2}(t)\right) \perp \cdots \perp\left(\bar{x}_{n+1}(t)-\right.$ $\left.\bar{x}_{n}(t)\right)$, and it follows for $m>n$ that

$$
\begin{aligned}
& \left\|\underline{x}_{m}(t)-\underline{x}_{n}(t)\right\|_{W_{2}^{1}}^{2}=\| \underline{x}_{m}(t)-\underline{x}_{m-1}(t)+\underline{x}_{m-1}(t) \\
& -\cdots+\underline{x}_{n+1}(t)-\underline{x}_{n}(t)\left\|_{W_{2}^{1}}^{2} \leq\right\| \underline{x}_{m}(t) \\
& -\underline{x}_{m-1}(t)\left\|_{W_{2}^{1}}^{2}+\cdots+\right\| \underline{x}_{n+1}(t)-\underline{x}_{n}(t) \|_{W_{2}^{1}}^{2} \\
& =\sum_{i=n+1}^{m}\left(A_{i}\right)^{2} \longrightarrow 0, \quad(n, m \longrightarrow \infty), \\
& \left\|\bar{x}_{m}(t)-\bar{x}_{n}(t)\right\|_{W_{2}^{1}}^{2}=\| \bar{x}_{m}(t)-\bar{x}_{m-1}(t)+\bar{x}_{m-1}(t) \\
& -\cdots+\bar{x}_{n+1}(t)-\bar{x}_{n}(t)\left\|_{W_{2}^{1}}^{2} \leq\right\| \bar{x}_{m}(t) \\
& -\bar{x}_{m-1}(t)\left\|_{W_{2}^{1}}^{2}+\cdots+\right\| \bar{x}_{n+1}(t)-\bar{x}_{n}(t) \|_{W_{2}^{1}}^{2} \\
& =\sum_{i=n+1}^{m}\left(B_{i}\right)^{2} \longrightarrow 0, \quad(n, m \longrightarrow \infty) .
\end{aligned}
$$

Considering the completeness of $W_{2}^{1}[a, b]$, there exist $\underline{x}(t)$ and $\bar{x}(t) \in W_{2}^{1}[a, b]$ such that $\underline{x}_{n}(t) \rightarrow \underline{x}(t)$ and $\bar{x}_{n}(t) \rightarrow \overline{\bar{x}}(t)$ as $n \rightarrow \infty$ in sense of $\|\cdot\|_{W_{2}^{1}}$.

Secondly, we will prove that $\underline{x}(t)$ and $\bar{x}(t)$ are the solutions to system (13). By Lemma 14 and since $\left\{t_{i}\right\}_{i=1}^{\infty}$ is dense on $[a, b]$, we know that $\underline{x}_{n}(t)$ and $\bar{x}_{n}(t)$ converge uniformly to $\underline{x}(t)$ and $\bar{x}(t)$, respectively. Taking limits in (18), one can get $\underline{x}(t)=\sum_{i=1}^{\infty} A_{i} \bar{\psi}_{i}(t)$ and $\bar{x}(t)=\sum_{i=1}^{\infty} B_{i} \bar{\psi}_{i}(t)$. Since

$$
\begin{aligned}
(L \underline{x})\left(t_{j}\right) & =\sum_{i=1}^{\infty} A_{i}\left\langle L \bar{\psi}_{i}(t), \Phi_{j}(t)\right\rangle_{W_{2}^{1}} \\
& =\sum_{i=1}^{\infty} A_{i}\left\langle\bar{\psi}_{i}(t), L^{*} \Phi_{j}(t)\right\rangle_{W_{2}^{1}} \\
& =\sum_{i=1}^{\infty} A_{i}\left\langle\bar{\psi}_{i}(t), \psi_{j}(t)\right\rangle_{W_{2}^{1}}, \\
(L \bar{x})\left(t_{j}\right) & =\sum_{i=1}^{\infty} B_{i}\left\langle L \bar{\psi}_{i}(t), \Phi_{j}(t)\right\rangle_{W_{2}^{1}} \\
& =\sum_{i=1}^{\infty} B_{i}\left\langle\bar{\psi}_{i}(t), L^{*} \Phi_{j}(t)\right\rangle_{W_{2}^{1}} \\
& =\sum_{i=1}^{\infty} B_{i}\left\langle\bar{\psi}_{i}(t), \psi_{j}(t)\right\rangle_{W_{2}^{1}},
\end{aligned}
$$

it follows that

$$
\begin{aligned}
\sum_{j=1}^{n} \beta_{n j}(L \underline{x})\left(t_{j}\right) & =\sum_{i=1}^{\infty} A_{i}\left\langle\bar{\psi}_{i}(t), \sum_{j=1}^{n} \beta_{n j} \psi_{j}(t)\right\rangle_{W_{2}^{1}} \\
& =\sum_{i=1}^{\infty} A_{i}\left\langle\bar{\psi}_{i}(t), \bar{\psi}_{n}(t)\right\rangle_{W_{2}^{1}}=A_{n}, \\
\sum_{j=1}^{n} \beta_{n j}(L \bar{x})\left(t_{j}\right) & =\sum_{i=1}^{\infty} B_{i}\left\langle\bar{\psi}_{i}(t), \sum_{j=1}^{n} \beta_{n j} \psi_{j}(t)\right\rangle_{W_{2}^{1}} \\
& =\sum_{i=1}^{\infty} B_{i}\left\langle\bar{\psi}_{i}(t), \bar{\psi}_{n}(t)\right\rangle_{W_{2}^{1}}=B_{n} .
\end{aligned}
$$

Now, if $n=1$, then $(L \underline{x})\left(t_{1}\right)=F\left(t_{1}, f\left(t_{1}\right), T \underline{x}_{0}\left(t_{1}\right), T \bar{x}_{0}\left(t_{1}\right)\right)$ and $(L \bar{x})\left(t_{1}\right)=G\left(t_{1}, \bar{f}\left(t_{1}\right), T \underline{x}_{0}\left(t_{1}\right), T \bar{x}_{0}\left(t_{1}\right)\right)$. Again, if $n=2$, then $(L \underline{x})\left(t_{2}\right)=F\left(t_{2}, \underline{f}\left(t_{2}\right), T \underline{x}_{1}\left(t_{2}\right), T \bar{x}_{1}\left(t_{2}\right)\right)$ and $(L \bar{x})\left(t_{2}\right)=$ $G\left(t_{2}, \bar{f}\left(t_{2}\right), T \underline{x}_{1}\left(t_{2}\right), T \bar{x}_{1}\left(t_{2}\right)\right)$. In the same way, it is easy to see by induction that $(L \underline{x})\left(t_{j}\right)=F\left(t_{j}, \underline{f}\left(t_{j}\right), T \underline{x}_{j-1}\left(t_{j}\right), T \bar{x}_{j-1}\left(t_{j}\right)\right)$ and $(L \bar{x})\left(t_{j}\right)=G\left(t_{j}, \bar{f}\left(t_{j}\right), T \underline{x}_{j-1}\left(t_{j}\right), T \bar{x}_{j-1}\left(t_{j}\right)\right)$. Since $\left\{t_{i}\right\}_{i=1}^{\infty}$ is dense on $[a, b]$, then, for any $y \in[a, b]$, there exists subsequence $\left\{t_{n_{j}}\right\}$ such that $t_{n_{j}} \rightarrow y$ as $j \rightarrow$ $\infty$. But, on the other aspect as well, we have known that $(L \underline{x})\left(t_{n_{j}}\right)=F\left(t_{n_{j}}, \underline{f}\left(t_{n_{j}}\right), T \underline{x}_{j-1}\left(t_{n_{j}}\right), T \bar{x}_{j-1}\left(t_{n_{j}}\right)\right)$ and $(L \bar{x})\left(t_{n_{j}}\right)=G\left(t_{n_{j}}, \bar{f}\left(t_{n_{j}}\right), T \underline{x}_{j-1}\left(t_{n_{j}}\right), T \bar{x}_{j-1}\left(t_{n_{j}}\right)\right)$. Hence, let $j \rightarrow \infty$, and, by the continuity of $F$ and $G$, we have $(L \underline{x})(y)=F(y, \underline{f}(y), T \underline{x}(y), T \bar{x}(y))$ and $(L \bar{x})(y)=G(y$, $\bar{f}(y), T \underline{x}(y), T \bar{x}(y))$. That is, $\underline{x}(t)$ and $\bar{x}(t)$ are the solutions to system (13). So, the proof of the theorem is complete.

The reliability of the numerical result will depend on an error estimate; therefore, the analysis of error and as well as the sources of error in numerical methods is also a critically important part of the study of numerical technique. 
Theorem 16. Assume that $\underline{x}(t)$ and $\bar{x}(t) \in W_{2}^{1}[a, b]$ are given by (16) and (17) and $r_{n}$ and $e_{n}$ are the errors in the approximate solutions $\underline{x}_{n}(t)$ and $\bar{x}_{n}(t)$, respectively, where $\underline{x}_{n}(t)$ and $\bar{x}_{n}(t)$ are given in (18). Then, the errors are monotone decreasing in the sense of $\|\cdot\|_{W_{2}^{1}}$.

Proof. Based on the previous results, it is obvious that

$$
\begin{aligned}
& \left\|r_{n}(t)\right\|_{W_{2}^{1}}^{2}=\left\|\underline{x}(t)-\underline{x}_{n}(t)\right\|_{W_{2}^{1}}^{2} \\
& =\| \sum_{i=n+1}^{\infty} \sum_{k=1}^{i} \beta_{i k} F\left(t_{k}, \underline{f}\left(t_{k}\right), T \underline{x}_{k-1}\left(t_{k}\right), T \bar{x}_{k-1}\left(t_{k}\right)\right) \\
& \cdot \bar{\psi}_{i}(t)\left\|_{W_{2}^{1}}^{2}=\right\| \sum_{i=n+1}^{\infty} A_{i} \bar{\psi}_{i}(t) \|_{W_{2}^{1}}^{2}=\sum_{i=n+1}^{\infty}\left(A_{i}\right)^{2}
\end{aligned}
$$

and $\left\|r_{n-1}(t)\right\|_{W_{2}^{1}}^{2}=\sum_{i=n}^{\infty}\left(A_{i}\right)^{2}$. Clearly, $\left\|r_{n}(t)\right\|_{W_{2}^{1}} \leq\left\|r_{n-1}(t)\right\|_{W_{2}^{1}}$. Consequently, the error $r_{n}$ is monotone decreasing in the sense of $\|\cdot\|_{W_{2}^{1}}$. Similarly, the error $e_{n}$ is monotone decreasing in the sense of $\|\cdot\|_{W_{2}^{1}}$. So, the proof of the theorem is complete.

Software packages have great capabilities for solving mathematical, physical, and engineering problems. The aim of the next algorithm is to implement a procedure to solve FIE (1) in numeric form in terms of its grid nodes based on the use of RKHS method.

Algorithm 17. To approximate the solution to system (5), we do the following steps.

Input. Consider the endpoints of $[0,1]$, the integer $n$, the kernel function $R_{t}(s)$, the operator $L$, and the functions $F$ and G.

Output. Consider approximate solutions $\underline{x}_{n}(t)$ and $\bar{x}_{n}(t)$.

Step 1. Fix $t$ in $[0,1]$ and set $s \in[0,1]$ :

If $s \leq t$, set $R_{t}(s)=1+s$.

Else set $R_{t}(s)=1+t$.

For $i=1,2, \ldots, n$, do the following.

Set $t_{i}=(i-1) /(n-1)$.

Set $\psi_{i}(t)=L_{s} R_{t}(s)_{\left.\right|_{s=t_{i}}}$.

Output is the orthogonal function system $\psi_{i}(t)$.

Step 2. For $i=1,2, \ldots, n$ and $j=1,2, \ldots, i$, do the following:

$$
\begin{aligned}
& \text { If } i \quad \neq \quad j, \text { then set } \beta_{i j}=\quad(-1 / \\
& \sqrt{\left.\left\|\psi_{i}\right\|^{2}-\sum_{k=1}^{i-1}\left\langle\psi_{i}(t), \bar{\psi}_{k}(t)\right\rangle^{2}\right)} \sum_{k=j}^{i-1}\left\langle\psi_{i}(t), \bar{\psi}_{k}(t)\right\rangle \beta_{k j} . \\
& \text { Else set } \beta_{i j}=1 / \sqrt{\left\|\psi_{i}\right\|^{2}-\sum_{k=1}^{i-1}\left\langle\psi_{i}(t), \bar{\psi}_{k}(t)\right\rangle^{2}} . \\
& \text { Else set } \beta_{11}=1 /\left\|\psi_{1}\right\| \text {. } \\
& \text { Output is the orthogonalization coefficients } \beta_{i j} .
\end{aligned}
$$

Step 3. For $i=2,3, \ldots, n-1$, do the following:
Set $\bar{\psi}_{i}(t)=\sum_{k=1}^{i} \beta_{i k} \psi_{k}(t)$

Output is the orthonormal function system $\bar{\psi}_{i}(t)$.

Step 4 . For $i=1,2, \ldots, n$, do the following:

$$
\begin{aligned}
& \text { Set } \underline{x}_{0}\left(t_{1}\right)=0 . \\
& \text { Set } \bar{x}_{0}\left(t_{1}\right)=0 . \\
& \text { Set } A_{i}=\sum_{k=1}^{i} \beta_{i k} F\left(t_{k}, \underline{f}\left(t_{k}\right), T \underline{x}_{k-1}\left(t_{k}\right), T \bar{x}_{k-1}\left(t_{k}\right)\right) . \\
& \text { Set } B_{i}=\sum_{k=1}^{i} \beta_{i k} G\left(t_{k}, \bar{f}\left(t_{k}\right), T \underline{x}_{k-1}\left(t_{k}\right), T \bar{x}_{k-1}\left(t_{k}\right)\right) . \\
& \text { Set } \underline{x}_{n}\left(t_{i}\right)=\sum_{i=1}^{n} A_{i} \bar{\psi}_{i}\left(t_{i}\right) . \\
& \text { Set } \bar{x}_{n}\left(t_{i}\right)=\sum_{i=1}^{n} B_{i} \bar{\psi}_{i}\left(t_{i}\right) .
\end{aligned}
$$

Output is the approximate solutions $\underline{x}_{n}\left(t_{i}\right)$ and $\bar{x}_{n}\left(t_{i}\right)$ and then stop.

\section{Experiment Results}

The proposed method provides analytical as well approximate solutions in terms of a rapidly convergent series with easily computable components. However, there is a practical need to evaluate these solutions and to obtain numerical values from these series. The consequent series truncation and the practical procedure are conducted to accomplish this task. In this section, we consider three examples to illustrate the efficiency and performance of the RKHS in finding approximate series solution for both linear and nonlinear FIEs. On the other hand, results obtained are compared with the exact solution to each example and are found to be in good agreement with each other. In the process of computation, all symbolic and numerical computations are performed by Mathematica 7.0 software package.

Example 1. Consider the following fuzzy Volterra integral equation:

$$
x(t)=[\alpha, 2-\alpha] t+\int_{0}^{t} e^{t-s} x(s) d s,
$$

where $0 \leq t \leq 1$. The exact fuzzy solution is $x(t)=$ $\left[(\alpha / 4)\left(e^{2 t}+2 t-1\right),((2-\alpha) / 4)\left(e^{2 t}+2 t-1\right)\right], \alpha \in[0,1]$.

For the conduct of proceedings in the fuzzy solution, we have the following system of CIEs taking into account that the crisp kernel function $k(t, s)=e^{t-s}$ is positive on $0 \leq t \leq$ 1 . Thus, by considering the parametric form of (27), one can write

$$
\begin{aligned}
& \underline{x}(t)=\alpha t+\int_{0}^{t} e^{t-s} \underline{x}(s) d s, \quad 0 \leq t \leq 1, \\
& \bar{x}(t)=(2-\alpha) t+\int_{0}^{t} e^{t-s} \bar{x}(s) d s, \quad 0 \leq t \leq 1,
\end{aligned}
$$

To illustrate the fuzzy behaviors of the approximate solutions at some specific certain computed nodes, the absolute errors of numerically approximating $x_{n}\left(t_{i}\right), n=101, t_{i}=$ $(i-1) /(n-1), i=1,2, \ldots, n$, for the corresponding CIE system have been calculated for various $\alpha$ and $t$ on $[0,1]$ as shown in Tables 1 and 2 . Here, the absolute errors in Table 1 are 


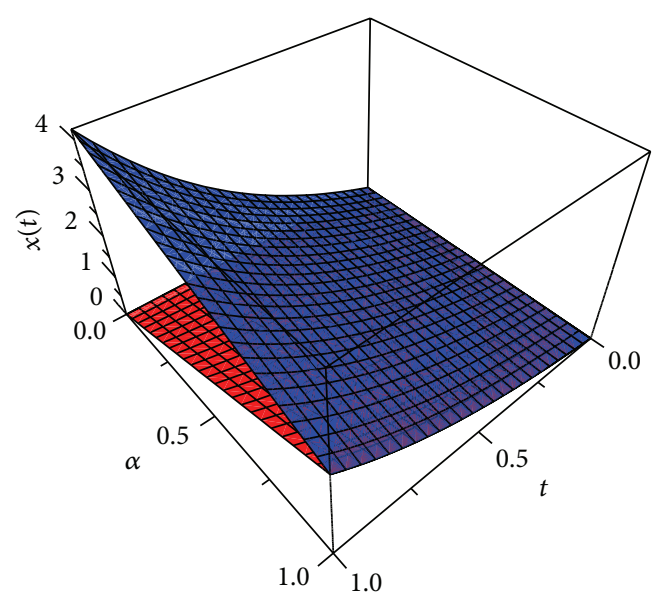

FIGURE 1: The fuzzy solution to (27) in parametric form, red $x(t)$ and blue $\bar{x}(t)$.

given by $e_{i}=\left|\underline{x}\left(t_{i}\right)-\underline{x}_{n}\left(t_{i}\right)\right|$ while in Table 2 they are given by $e_{i}=\left|\bar{x}\left(t_{i}\right)-\bar{x}_{n}\left(t_{i}\right)\right|$. Anyhow, it is clear from the tables that the approximate solutions are in close agreement with the exact solutions as well; we see that $\underline{x}(t)=\bar{x}(t)$ at $\alpha=1$. The fuzzy solution to (27) has been plotted as shown in Figure 1. On the other aspect as well, one can see that the numerical result satisfies the convex symmetric triangular fuzzy number.

Example 2. Consider the following fuzzy Volterra integral equation:

$$
x(t)=[\alpha-1,1-\alpha] t-\int_{0}^{t} x(s) d s
$$

where $0 \leq t \leq 1$. The exact fuzzy solution is $x(t)=[(\alpha-$ $1)(\sinh (t)+\cosh (t)-1),(1-\alpha)(\sinh (t)+\cosh (t)-1)], \alpha \in$ $[0,1]$.

Using the RKHS method, taking $t_{i}=(i-1) /(n-1), i=$ $1,2, \ldots, n$, if we choose negative crisp kernel function $k(t, s)=$ -1 on $0 \leq t \leq 1$, then the corresponding crisp IE system of (29) can be written as

$$
\begin{aligned}
& \underline{x}(t)=(\alpha-1) t-\int_{0}^{t} \bar{x}(s) d s, \quad 0 \leq t \leq 1, \\
& \bar{x}(t)=(1-\alpha) t-\int_{0}^{t} \underline{x}(s) d s, \quad 0 \leq t \leq 1 .
\end{aligned}
$$

The numerical results for the corresponding crisp IE system of (29) for $\alpha=0.75$ and various $t$ in $[0,1]$ are given in Tables 3 and 4 . As we mentioned earlier, it is possible to pick any point in the independent interval of $t$ and as well the approximate solutions will be applicable. The fuzzy solution to (29) has been plotted as shown in Figure 2.

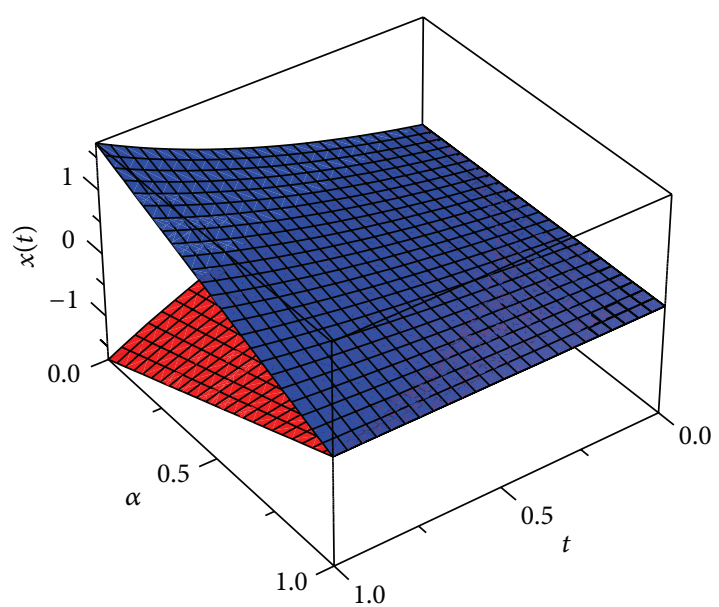

FIGURE 2: The fuzzy solution to FIE (29) in parametric form, red $\underline{x}(t)$ and blue $\bar{x}(t)$.

Example 3. Consider the following nonlinear fuzzy Volterra integral equation:

$$
x(t)=[\alpha, 2-\alpha]+\int_{0}^{t} \sqrt{x(s)} d s,
$$

where $0 \leq t \leq 1$. The exact solution is $x(t)=[((1 / 2) t+$ $\left.\sqrt{\alpha})^{2},((1 / 2) t+\sqrt{2-\alpha})^{2}\right], \alpha \in[0,1]$.

Here, it can be observed that $g(s)=\sqrt{s}$ is a continuous increasing function on $[0, \infty)$. Then, by using Zadeh's extension principle, we get $[g(x(s))]_{\alpha}=[\sqrt{\underline{x}(s)}, \sqrt{\bar{x}(s)}]$ for all $\alpha \in[0,1]$. Furthermore, the crisp kernel function $k(t, s)$ is positive on $0 \leq t \leq 1$. So, the FIE (31) can be converted into nonlinear crisp integral equations system as

$$
\begin{aligned}
& \underline{x}(t)=\alpha+\int_{0}^{t} \sqrt{\underline{x}(s)} d s, \quad 0 \leq t \leq 1, \\
& \bar{x}(t)=(2-\alpha)+\int_{0}^{t} \sqrt{\bar{x}(s)} d s, \quad 0 \leq t \leq 1 .
\end{aligned}
$$

The absolute errors of numerically approximating $\underline{x}(t)$ and $\bar{x}(t)$ for the corresponding crisp integral equations system (32) have been calculated at $\alpha=0.5$ and various $t$ as shown in Tables 5 and 6 . It is clear from the tables that the approximate solutions are in close agreement with the analytic solutions, by using only $n=101$ in our algorithm. Indeed, we can conclude that higher accuracy can be achieved by computing further RKHS steps for this nonlinear example. Finally, the fuzzy solution to FIE (31) has been plotted as shown in Figure 3.

\section{Conclusion}

The aim of present analysis is to propose a relatively recent numerical method for solving a class of fuzzy Volterra integral equations using the concept of Riemann integrability. The method is applied directly without using linearization, transformation, or restrictive conditions. Numerical results 
TABLE 1: The absolute errors of approximating $\underline{x}(t)$ for (27) at various $\alpha$ and $t$.

\begin{tabular}{lcccc}
\hline$t_{i}$ & $\alpha=0.25$ & $\alpha=0.5$ & $\alpha=0.75$ & $\alpha=1$ \\
\hline 0.25 & $3.17558 \times 10^{-7}$ & $6.35116 \times 10^{-7}$ & $9.52673 \times 10^{-7}$ & $1.27023 \times 10^{-6}$ \\
0.5 & $6.97419 \times 10^{-7}$ & $1.39484 \times 10^{-6}$ & $2.09226 \times 10^{-6}$ & $2.78968 \times 10^{-6}$ \\
0.75 & $8.61564 \times 10^{-7}$ & $1.72313 \times 10^{-6}$ & $2.58469 \times 10^{-6}$ & $3.44626 \times 10^{-6}$ \\
1 & $4.44089 \times 10^{-15}$ & $8.88178 \times 10^{-16}$ & $8.43769 \times 10^{-15}$ & $1.77636 \times 10^{-15}$ \\
\hline
\end{tabular}

TABLE 2: The absolute errors of approximating $\bar{x}(t)$ for (27) at various $\alpha$ and $t$.

\begin{tabular}{lcccc}
\hline$t_{i}$ & $\alpha=0.25$ & $\alpha=0.5$ & $\alpha=0.75$ & $\alpha=1$ \\
\hline 0.25 & $2.22290 \times 10^{-6}$ & $1.90535 \times 10^{-6}$ & $1.58779 \times 10^{-6}$ & $1.27023 \times 10^{-6}$ \\
0.5 & $4.88193 \times 10^{-6}$ & $4.18452 \times 10^{-6}$ & $3.4871 \times 10^{-6}$ & $2.78968 \times 10^{-6}$ \\
0.75 & $6.03095 \times 10^{-6}$ & $5.16939 \times 10^{-6}$ & $4.30782 \times 10^{-6}$ & $3.44626 \times 10^{-6}$ \\
1 & $7.10543 \times 10^{-15}$ & $1.68754 \times 10^{-14}$ & $1.28786 \times 10^{-14}$ & $1.77636 \times 10^{-15}$ \\
\hline
\end{tabular}

TABLE 3: The numerical results of $\underline{x}(t)$ for (29) at $\alpha=0.75$ and various $t$.

\begin{tabular}{lccc}
\hline$t_{i}$ & Exact solution & Approximate solution & Absolute error \\
\hline 0.16 & 0.13351087099181003 & 0.1335113660587801 & $4.9506697008538940 \times 10^{-7}$ \\
0.36 & 0.29712776433595710 & 0.2971287051998124 & $9.4086385526992440 \times 10^{-7}$ \\
0.48 & 0.49607440219289334 & 0.4960756690151702 & $1.2668222768574822 \times 10^{-6}$ \\
0.64 & 0.73648087930495150 & 0.7364822519454437 & $1.3726404921587942 \times 10^{-6}$ \\
0.80 & 1.02554092849246800 & 1.0255420474086065 & $1.1189161386315760 \times 10^{-6}$ \\
0.96 & 1.37169647342311780 & 1.3716967886418380 & $3.1521872023709820 \times 10^{-7}$ \\
\hline
\end{tabular}

TABLE 4: The numerical results of $\bar{x}(t)$ for (29) at $\alpha=0.75$ and various $t$.

\begin{tabular}{lccc}
\hline$t_{i}$ & Exact solution & Approximate solution & Absolute error \\
\hline 0.16 & 0.21351087099181010 & 0.21351136605878263 & $4.9506697252788000 \times 10^{-7}$ \\
0.32 & 0.45712776433595714 & 0.45712870519981497 & $9.4086385782343740 \times 10^{-7}$ \\
0.48 & 0.73607440219289340 & 0.73607566901517440 & $1.2668222809653074 \times 10^{-6}$ \\
0.64 & 1.05648087930495160 & 1.05648225194544980 & $1.3726404981539986 \times 10^{-6}$ \\
0.80 & 1.42554092849246780 & 1.42554204740861000 & $1.1189161421842897 \times 10^{-6}$ \\
0.96 & 1.85169647342311780 & 1.85169678864184250 & $3.1521872467799030 \times 10^{-7}$ \\
\hline
\end{tabular}

TABLE 5: The numerical results of $\underline{x}(t)$ for (31) at $\alpha=0.5$ and various $t$.

\begin{tabular}{lccc}
\hline$t_{i}$ & Exact solution & Approximate solution & Absolute error \\
\hline 0.16 & 0.6195370849898476 & 0.6195372960438028 & $2.1105395520137904 \times 10^{-7}$ \\
0.32 & 0.7518741699796954 & 0.751874511686097 & $3.4170640161867993 \times 10^{-7}$ \\
0.48 & 0.8970112549695429 & 0.897011646926886 & $3.9195734302666096 \times 10^{-7}$ \\
0.64 & 1.0549483399593904 & 1.0549487017661694 & $3.618067789812329 \times 10^{-7}$ \\
0.80 & 1.2256854249492382 & 1.2256856762039468 & $2.512547085942174 \times 10^{-7}$ \\
0.96 & 1.4092225099390854 & 1.4092225702402188 & $6.03011334199266 \times 10^{-8}$ \\
\hline
\end{tabular}

TABLE 6: The numerical results of $\bar{x}(t)$ for (31) at $\alpha=0.5$ and various $t$.

\begin{tabular}{lccc}
\hline$t_{i}$ & Exact solution & Approximate solution & Absolute error \\
\hline 0.16 & 1.7023591794226542 & 1.70235939047661 & $2.1105395564546825 \times 10^{-7}$ \\
0.32 & 1.917518358845308 & 1.9175187005517114 & $3.4170640339503677 \times 10^{-7}$ \\
0.48 & 2.1454775382679623 & 2.145477930225306 & $3.919573439148394 \times 10^{-7}$ \\
0.64 & 2.386236717690617 & 2.386237079497394 & $3.618067769828315 \times 10^{-7}$ \\
0.80 & 2.6397958971132707 & 2.6397961483679766 & $2.5125470592968213 \times 10^{-7}$ \\
0.96 & 2.906155076535925 & 2.906155136837056 & $6.030113075539134 \times 10^{-8}$ \\
\hline
\end{tabular}




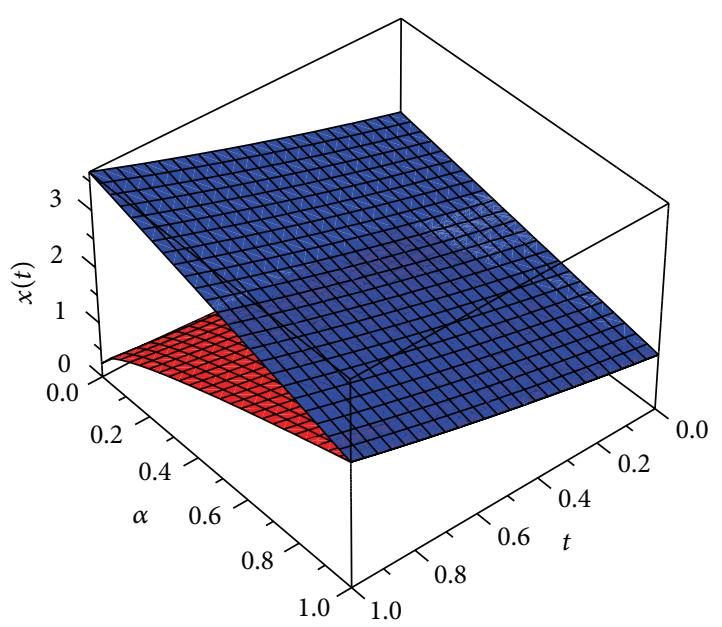

FIgURE 3: The fuzzy solution to FIE (31) in parametric form, red $\underline{x}(t)$ and blue $\bar{x}(t)$.

show that the RKHS is of higher precision and powerful technique and applicable to solve different types of fuzzy integral equations. Moreover, the accuracy of the solution can be improved by selecting large value of $n$.

\section{Competing Interests}

The authors declare that there is no conflict of interests regarding the publication of this paper.

\section{References}

[1] M. Hanss, Applied Fuzzy Arithmetic: An Introduction with Engineering Applications, Springer, Berlin, Germany, 2005.

[2] E. Hüllermeier, "An approach to modelling and simulation of uncertain dynamical systems," International Journal of Uncertainty, Fuzziness and Knowledge-Based Systems, vol. 5, no. 2, pp. 117-137, 1997.

[3] S. Barro and R. Marın, Fuzzy Logic in Medicine, Physica, Heidelberg, Germany, 2002.

[4] M. Mosleh, "Fuzzy neural network for solving a system of fuzzy differential equations," Applied Soft Computing, vol. 13, no. 8, pp. 3597-3607, 2013.

[5] M. Guo, X. Xue, and R. Li, "Impulsive functional differential inclusions and fuzzy population models," Fuzzy Sets and Systems, vol. 138, no. 3, pp. 601-615, 2003.

[6] H. Zhang, X. Liao, and J. Yu, "Fuzzy modeling and synchronization of hyperchaotic systems," Chaos, Solitons and Fractals, vol. 26, no. 3, pp. 835-843, 2005.

[7] M. S. El Naschie, "From experimental quantum optics to quantum gravity via a fuzzy Kähler manifold," Chaos, Solitons \& Fractals, vol. 25, no. 5, pp. 969-977, 2005.

[8] P. Diamond, "Time-dependent differential inclusions, cocycle attractors and fuzzy differential equations," IEEE Transactions on Fuzzy Systems, vol. 7, no. 6, pp. 734-740, 1999.

[9] G. Feng and G. Chen, "Adaptive control of discrete-time chaotic systems: a fuzzy control approach," Chaos, Solitons and Fractals, vol. 23, no. 2, pp. 459-467, 2005.

[10] A. Bencsik, B. Bede, J. Tar, and J. Fodor, "Fuzzy differential equations in modeling hydraulic differential servo cylinders," in Proceedings of the 3rd Romanian-Hungarian Joint Symposium on Applied Computational Intelligence (SACI '06), Timisoara, Romania, 2006.

[11] M. Ghanbari, "Numerical solution of fuzzy linear Volterra integral equations of the second kind by Homotopy analysis method," International Journal Industrial Mathematics, vol. 2, pp. 73-87, 2010.

[12] S. Abbasbandy and T. Allahviranloo, "The Adomian decomposition method applied to the fuzzy system of Fredholm integral equations of the second kind," International Journal of Uncertainty, Fuzziness and Knowledge-Based Systems, vol. 14, no. 1, pp. 101-110, 2006.

[13] H. Attari and A. Yazdani, "A computational method for fuzzy Volterra-Fredholm integral equations," Fuzzy Information Engineering, vol. 2, pp. 147-156, 2011.

[14] M. A. Faborzi Araghi and N. Parandin, "Numerical solution of fuzzy Fredholm integral equations by the Lagrange interpolation based on the extension principle," Soft Computing, vol. 15, no. 12, pp. 2449-2456, 2011.

[15] S. Salahshour and T. Allahviranloo, "Application of fuzzy differential transform method for solving fuzzy Volterra integral equations," Applied Mathematical Modelling, vol. 37, no. 3, pp. 1016-1027, 2013.

[16] S. Salahshour, M. Khezerloo, S. Hajighasemi, and M. Khorasany, "Solving fuzzy integral equations of the second kind by fuzzy Laplace transform method," International Journal Industrial Mathematics, vol. 1, pp. 21-29, 2012.

[17] A. M. Bica and C. Popescu, "Numerical solutions of the nonlinear fuzzy Hammerstein-Volterra delay integral equations," Information Sciences, vol. 223, pp. 236-255, 2013.

[18] J. Y. Park and H. K. Han, "Existence and uniqueness theorem for a solution of fuzzy Volterra integral equations," Fuzzy Sets and Systems, vol. 105, no. 3, pp. 481-488, 1999.

[19] F. Z. Geng, S. P. Qian, and M. G. Cui, "Improved reproducing kernel method for singularly perturbed differential-difference equations with boundary layer behavior," Applied Mathematics and Computation, vol. 252, pp. 58-63, 2015.

[20] O. A. Arqub, M. Al-Smadi, and N. Shawagfeh, "Solving Fredholm integro-differential equations using reproducing kernel Hilbert space method," Applied Mathematics and Computation, vol. 219, no. 17, pp. 8938-8948, 2013.

[21] I. Komashynska and M. AL-Smadi, "Iterative reproducing kernel method for solving second-order integrodifferential equations of Fredholm type," Journal of Applied Mathematics, vol. 2014, Article ID 459509, 11 pages, 2014.

[22] O. Abu Arqub, M. Al-Smadi, and S. Momani, "Application of reproducing kernel method for solving nonlinear FredholmVolterra integro-differential equations," Abstract and Applied Analysis, vol. 2012, Article ID 839836, 16 pages, 2012.

[23] M. Al-Smadi, O. Abu Arqub, and S. Momani, "A computational method for two-point boundary value problems of fourth-order mixed integrodifferential equations," Mathematical Problems in Engineering, vol. 2013, Article ID 832074, 10 pages, 2013.

[24] O. Abu Arqub and M. Al-Smadi, "Numerical algorithm for solving two-point, second-order periodic boundary value problems for mixed integro-differential equations," Applied Mathematics and Computation, vol. 243, pp. 911-922, 2014.

[25] M. Al-Smadi, O. Abu Arqub, and A. El-Ajou, "A numerical iterative method for solving systems of first-order periodic boundary value problems," Journal of Applied Mathematics, vol. 2014, Article ID 135465, 10 pages, 2014. 
[26] M. Al-Smadi, O. Abu Arqub, and N. Shawagfeh, "Approximate solution of BVPs for 4th-order IDEs by using RKHS method," Applied Mathematical Sciences, vol. 6, no. 50, pp. 2453-2464, 2012.

[27] R. Goetschel Jr. and W. Voxman, "Elementary fuzzy calculus," Fuzzy Sets and Systems, vol. 18, no. 1, pp. 31-43, 1986.

[28] O. Kaleva, "Fuzzy differential equations," Fuzzy Sets and Systems, vol. 24, no. 3, pp. 301-317, 1987.

[29] O. Abu Arqub, M. Al-Smadi, S. Momani, and T. Hayat, "Numerical solutions of fuzzy differential equations using reproducing kernel Hilbert space method," Soft Computing, 2015.

[30] J. Y. Park and J. U. Jeong, "A note on fuzzy integral equations," Fuzzy Sets and Systems, vol. 108, no. 2, pp. 193-200, 1999.

[31] M. L. Puri and D. A. Ralescu, "Fuzzy random variables," Journal of Mathematical Analysis and Applications, vol. 114, no. 2, pp. 409-422, 1986.

[32] M. L. Puri and D. A. Ralescu, "Differentials of fuzzy functions," Journal of Mathematical Analysis and Applications, vol. 91, no. 2, pp. 552-558, 1983.

[33] F. Geng, "Solving singular second order three-point boundary value problems using reproducing kernel Hilbert space method," Applied Mathematics and Computation, vol. 215, no. 6, pp. 2095-2102, 2009.

[34] F. Geng, "A new reproducing kernel Hilbert space method for solving nonlinear fourth-order boundary value problems," Applied Mathematics and Computation, vol. 213, no. 1, pp. 163169, 2009. 


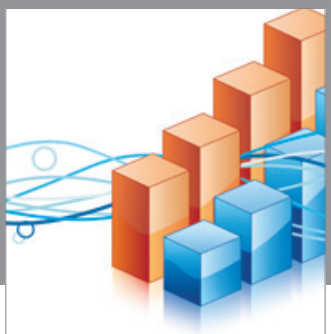

Advances in

Operations Research

vatem alat4

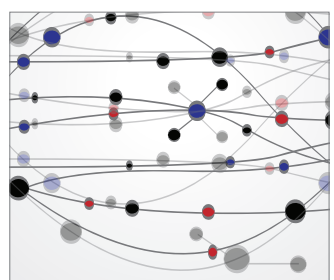

\section{The Scientific} World Journal
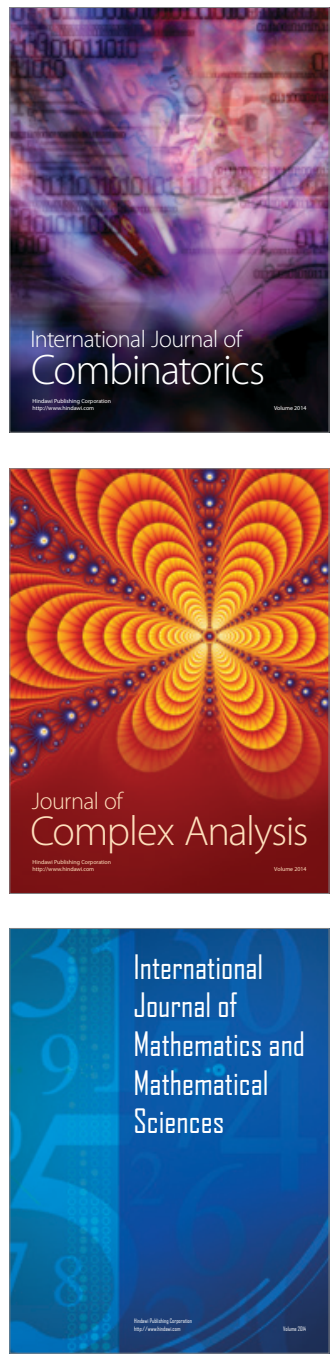
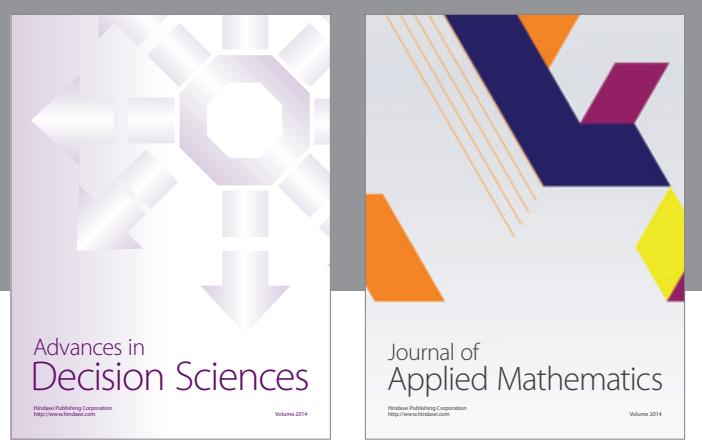

Algebra

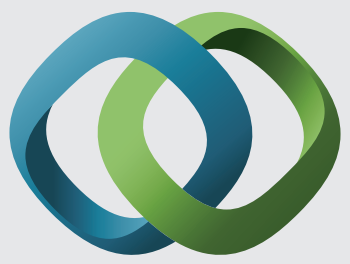

\section{Hindawi}

Submit your manuscripts at

http://www.hindawi.com
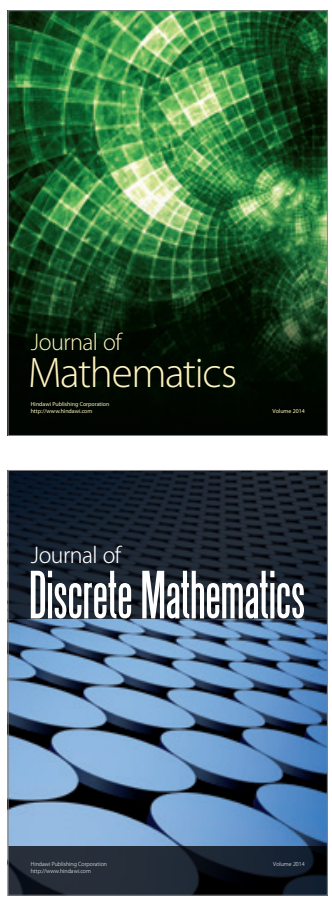

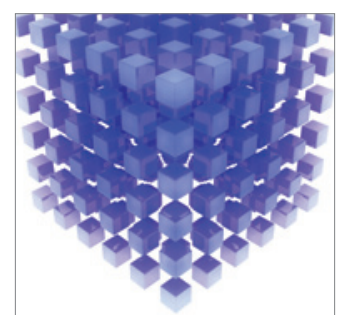

Mathematical Problems in Engineering
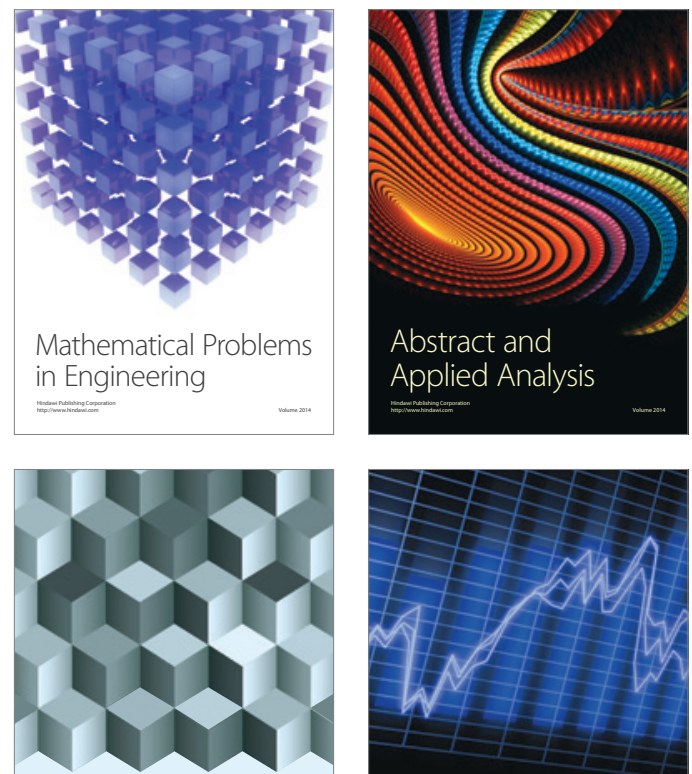

Journal of

Function Spaces

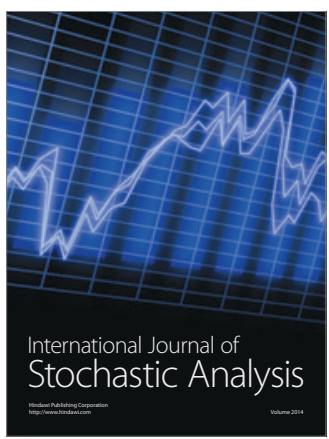

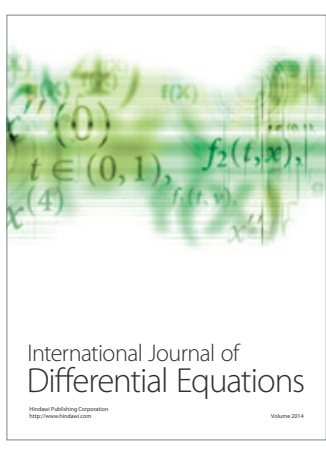
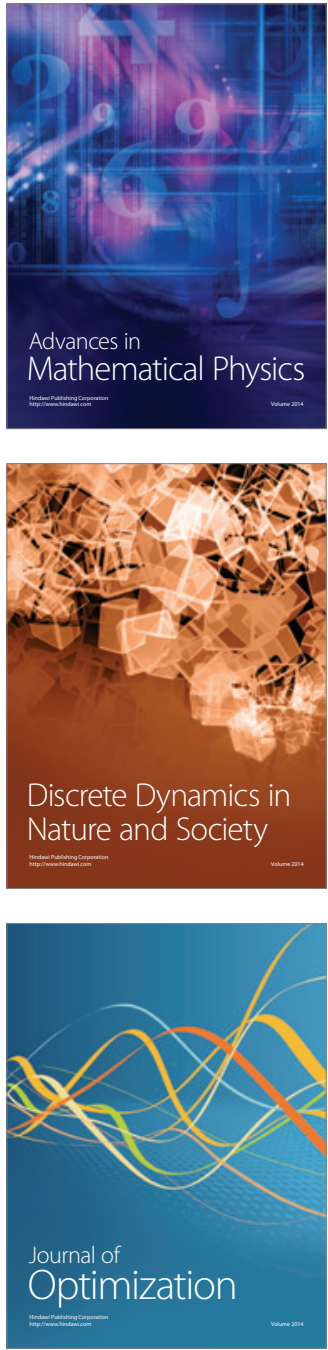